\title{
KNOTTING MANIFOLDS
}

\author{
BY E. C. ZEEMAN
}

Communicated by S. Eilenberg, September 7, 1960

We give a definition of isotopy and of knotting of an arbitrary space in an arbitrary space. The definition is of most interest when the spaces are manifolds. To avoid pathology, and to obtain theorems, we confine ourselves to combinatorial theory.

Definitions. Let $Y$ be a finite simplicial complex, and let $I$ denote the unit interval. An isotopy of $Y$ is a piecewise linear homeomorphism $h: I \times Y \rightarrow I \times Y$ such that

(i) $h(t \times Y)=t \times Y, 0 \leqq t \leqq 1$; (therefore for each $t$ there is a piecewise linear homeomorphism $h_{t}: Y \rightarrow Y$, such that $\left.h(t, y)=\left(t, h_{t} y\right)\right)$;

(ii) $h_{0}$ is the identity on $Y$.

If $X$ is another complex, two piecewise linear embeddings $f, g: X \rightarrow Y$ are isotopic if there is an isotopy $h$ of $Y$ such that $h_{1} f=g$. Isotopy is an equivalence relation on the set of all piecewise linear embeddings of $X$ in $Y$. Let Iso $(X \subset Y)$ denote the set of equivalence classes. There is a natural map from $\operatorname{Iso}(X \subset Y)$ onto $\operatorname{Hom}(X \subset Y)$, the set of homotopy classes of piecewise linear embeddings, because isotopic maps are clearly homotopic. If this map is one-to-one we say that $X$ unknots in $Y$; otherwise we say $X$ knots in $Y$.

Examples. (i) The circle knots in the 3-sphere, as is well known, because there are many isotopy classes but only one homotopy class.

(ii) A point unknots in a closed manifold, and knots in a bounded manifold, because there is one isotopy class for the interior of the manifold, and one for each boundary component. For the rest of this paper, however, we shall confine ourselves to closed manifolds. (There is a relative theory which is more appropriate for bounded manifolds.)

Motivation. We choose this definition of isotopy, because it seems the most natural intuitive concept associated with the notion of piecewise linear embedding. In effect, we say that two embeddings $f, g$ of $X$ in $Y$ are equivalent if we can, not only slide $f X$ onto $g X$, but slide the pair $f X \subset Y$ onto the pair $g X \subset Y$, through a continuous family of equivalent piecewise linear embeddings. Our definition is stronger than Mazur's ambient isotopy, since it involves the embeddings $f, g$ specifically (see $[6, \S \S 2,3]$ ). It is in fact the strongest available combinatorial definition of isotopy, and so Theorems 1 and 2 below are in their most powerful form. 
Mazur leaves the embeddings out of his definition of isotopy, because he is interested in adding knots in spheres. We put the embeddings into our definition because we wish to compare Iso $(X \subset Y)$ with Hom $(X \subset Y)$. Although Iso $(X \subset Y)$ is a more natural geometrical set than $\operatorname{Hom}(X \subset Y)$, the latter is better known because it is closely allied to the algebraic invariants of homology and homotopy. Therefore in order to bridge the gap between geometry and algebra it is desirable to know when Iso $(X \subset Y)=\operatorname{Hom}(X \subset Y)$, namely, when $X$ unknots in $Y$.

THEOREM 1. If $S^{n}, S^{k}$ are combinatorial spheres, then $S^{n}$ unknots in $S^{k}$, provided $k \geqq n+3$.

THEOREM 2. If $M^{n}, Q^{k}$ are connected closed combinatorial manifolds, then $M^{n}$ unknots in $Q^{k}$, provided that

(a) $k \geqq n+4$,

(b) $M^{n}$ is $(2 n-k+1)$-connected, and

(c) $Q^{k}$ is $(2 n-k+3)$-connected.

The proofs of Theorems 1 and 2 are long technical combinatorial arguments, and similar to those in $[7 ; 9]$. The proof of Theorem 1 depends upon an idea of John Stallings'. He himself has an analogous theorem, unknotting spheres in spheres, which is more general, and in particular unknots $S^{n}$ in $S^{n+2}$ when the complement is a homotopy $S^{1}$, but is a topological form of unknotting rather than a combinatorial unknotting (similar to his solution of the Poincaré conjecture [8]).

Theorem 1 is used in the proof of Theorem 2. However, we cannot use Theorem 1 to improve hypothesis (a) of Theorem 2 to include the case $k=n+3$, because, although the connectivity hypotheses (b) and (c) would then imply that $M^{n}$ and $Q^{k}$ were spheres when $n \geqq 5$ (see [8]), they would not necessarily be combinatorial spheres, but only combinatorial manifolds triangulating spheres.

To show that hypotheses (a), (b), and (c) of Theorem 2, or perhaps a weakened version of them, are necessary, we give the following counterexamples:

THEOREM 3. (a) $S^{n}$ knots in $S^{n+2}, n \geqq 1$.

(b) $S^{r} \times S^{q}$ knots in $S^{2 q+1}$, if $S^{q}$ admits a continuous $r$-field of tangent vectors.

(c) $S^{n}$ knots in $S^{1} \times S^{2 n}$.

In the proof of (a) the knot is constructed by Artin's method of spinning a 1-dimensional knot (see [2]), and is proved to be knotted 
by computing the fundamental group of the complement. In the proof of (b) the knot is constructed so that it contains two linked $q$ spheres in $S^{2 q+1}$. In the proof of (c) the knot is constructed by connecting two small linking $n$-spheres by a pipe running round the $S^{1}$, and is proved to be knotted by a theorem of Irwin [4], using homological linking in the universal covering space of $S^{1} \times S^{2 n}$.

Theorems 1 and 3(a) almost enable us to answer the question posed in [9], and say: the only euclidean space in which we can knot $S^{n}$ is $(n+2)$-dimensions. The single outstanding case is $(n+1)$-dimensions, because, although the Schoenflies-Mazur-Brown Theorem (see $[3 ; 5]$ ) gives an unknotting of $S^{n}$ in $S^{n+1}$, it is topological, rather than combinatorial in our sense.

To bring home the difference between knotting spheres and knotting other manifolds, we conclude by indulging in a few numbers to illustrate the theorems:

$S^{50}$ knots in $S^{52}$, unknots in $S^{53}$, but knots again in $S^{1} \times S^{100}$. $S^{1} \times S^{49}$ knots in $S^{99}$, and unknots in $S^{101}$.

\section{BiBLIOGRAPHY}

1. J. W. Alexander, The combinatorial theory of complexes, Ann. of Math. vol. 31 (1930) pp. 292-320.

2. E. Artin, Zur Isotopie zweidimensionalen Flächen im $R_{4}$, Abh. Math. Sem. Univ. Hamburg vol. 4 (1926) pp. 174-177.

3. M. Brown, A proof of the generalized Schoenflies theorem, Bull. Amer. Math. Soc. vol. 66 (1960) pp. 74-76.

4. M. C. Irwin, $A$ generalisation of Dehn's Lemma, to appear.

5. B. Mazur, On embeddings of spheres, Bull. Amer. Math. Soc. vol. 65 (1959) pp. 59-65.

6. - The definition of equivalence of combinatorial imbeddings, Inst. Hautes Etudes Sci. Publ. Math. vol. 3 (1959) pp. 5-17.

7. R. Penrose, J. H. C. Whitehead and E. C. Zeeman, Imbedding of manifolds in Euclidean space, Ann. of Math., to appear.

8. J. R. Stallings, Polyhedral homotopy-spheres, Bull. Amer. Math. Soc., to appear.

9. E. C. Zeeman, Unknotting spheres, Ann. of Math. vol. 72 (1960) pp. 350-361.

Gonville and Caius College, Cambridge, England. 